Neurosurg Focus 27 (6):E10, 2009

\title{
Stereotactic spine radiosurgery for intradural and intramedullary metastasis
}

\author{
Dong Ah Shin, M.D., ${ }^{1}$ Ryoong Huh, M.D., ${ }^{1}$ SAng Sup Chung, M.D., ${ }^{1}$ Jack Rock, M.D., ${ }^{2}$ \\ ANd Samuel RYu, M.D. ${ }^{2,3}$ \\ ${ }^{1}$ Department of Neurosurgery, CHA University, Pochon, Republic of Korea; \\ and Departments of ${ }^{3}$ Radiation Oncology and ${ }^{2}$ Neurosurgery, Henry Ford Hospital, Detroit, Michigan
}

\begin{abstract}
Object. Stereotactic radiosurgery (SRS) has become an important treatment alternative to surgery for a variety of spinal lesions. However, the use of SRS in the management of intradural intramedullary (IDIM) metastasis remains controversial. The aim of this study was to determine the clinical efficacy and safety of SRS for treatment of IDIM metastasis.

Methods. Nine patients with 11 IDIM metastases treated with SRS at Henry Ford Hospital were retrospectively reviewed. The mean age at presentation was 50 years, with a range of 14-71 years. There were 4 intradural extramedullary and 7 intramedullary lesions. The radiosurgery procedure used techniques of image-guided and intensitymodulated radiation. The mean treatment dose was $13.8 \mathrm{~Gy}$, with a range of 10-16 Gy. All patients had clinical follow-up (except in 1 lesion), with an emphasis on initial symptoms and ambulatory status, and 8 patients (9 lesions) had imaging studies. The median follow-up duration was 10 months.

Results. The presenting symptoms were improved in $8(80 \%)$ of 10 evaluable lesions, unchanged in 1 case, and worsened in 1 case. Radiographic responses were seen as follows: complete response in $2(22 \%)$ of 9 ; partial response in $3(33 \%)$ of 9 ; stable disease in $3(33 \%)$ of 9 ; and progressive disease in $1(11 \%)$ of 9 . After radiosurgery, 7 patients $(78 \%)$ remained ambulatory until the last follow-up visit. The overall median survival time after SRS was 8 months, with a range of 2-19 months. No radiation toxicity was detected clinically during the follow-up period.

Conclusions. Despite the fact that this was a small series of patients with IDIM metastasis who had limited treatment options, SRS appears to be an effective and safe method of treating patients with these lesions. (DOI: 10.3171/2009.9.FOCUS09194)
\end{abstract}

\section{KeY WoRds • radiosurgery - spinal cord neoplasm • intramedullary spinal cord lesion • metastasis}

$I^{2}$ NTRADURAL and intramedullary metastasis consists of leptomeningeal and intramedullary metastasis. Whereas metastatic involvement is most common among the tumors arising from the spine, leptomeningeal metastasis is relatively unusual; it is observed in $4 \%$ of all spinal metastases. ${ }^{18}$ Intramedullary metastasis is even rarer, and affects only $0.1-0.4 \%$ of all cancer patients. ${ }^{3}$ Once the disease is diagnosed, the treatment with conventional radiation and surgery is limited and these patients have a poor prognosis. ${ }^{3,18}$ Despite their rarity and poor prognosis, the lesions are clinically more often detected with the use of MR imaging, and therapeutic advances with radiation, surgery, and chemotherapy may help improve the clinical outcome.

Stereotactic radiosurgery has become an important treatment alternative for a variety of spinal lesions due to its noninvasiveness and convenience..$^{1,4,5,12,14,16,19}$ Instead

\footnotetext{
Abbreviations used in this paper: $\mathrm{EBRT}=$ external-beam radiation therapy; IDIM = intradural intramedullary; IMRT = intensitymodulated radiation therapy; $\mathrm{SRS}=$ stereotactic radiosurgery.
}

of using multiple fractionations as in conventional radiotherapy, SRS can minimize radiation to the healthy tissue by using multiple beams, a high degree of collimation, and immobilization. ${ }^{14,16}$ The technical advances allow the use of single or a few fractionations, adding convenience to treatments for these seriously ill patients. The usefulness of SRS for spinal epidural lesions has been reported by many investigators. ${ }^{1,4,5,12,14,16,19}$ However, the use of SRS in the management of IDIM remains unknown. In the present study, we conducted a retrospective evaluation of our experience with SRS in 9 patients with IDIM to evaluate its clinical efficacy and safety.

\section{Methods}

\section{Patient Population}

The medical records of 9 patients with IDIM metastasis who underwent SRS at the Radiosurgery Center, Henry Ford Hospital, were retrospectively analyzed. Patients were consecutively treated with SRS over a 7-year period between November 2001 and September 2008. 
TABLE 1: Characteristics and clinical profiles in 9 patients with IDIM metastases*

\begin{tabular}{|c|c|c|c|c|c|c|c|}
\hline $\begin{array}{l}\text { Case } \\
\text { No. }\end{array}$ & $\begin{array}{c}\text { Age } \\
\text { (yrs), } \\
\text { Sex }\end{array}$ & $\begin{array}{c}\text { Time } \\
\text { Since } \\
\text { Primary } \\
\text { Dx (mos) }\end{array}$ & Primary Cancer & Synchronous Metastasis & Level & $\begin{array}{l}\text { Loca- } \\
\text { tion }\end{array}$ & Presenting Sx \\
\hline 1 & $51, \mathrm{M}$ & 167 & skin, melanoma & brain & C-6 & IM & arm \& leg numbness \\
\hline \multirow[t]{2}{*}{2} & $36, M$ & 32 & brain, choroid plexus carcinoma & brain & $\mathrm{C}-6$ & IDEM & hemiparesis \\
\hline & & & & & C5-7, T4-6 & IDEM & hemiparesis \\
\hline 3 & $50, F$ & 32 & breast & brain & C-7 & $\mathrm{IM}$ & paraplegia \\
\hline 4 & $71, \mathrm{~F}$ & 33 & kidney, renal cell carcinoma & brain & C-3 & $\mathrm{IM}$ & paraparesis \& arm numbness \\
\hline 5 & $47, \mathrm{~F}$ & 51 & breast, invasive ductal cell carcinoma & brain & C-5 & $\mathrm{IM}$ & radicular pain \\
\hline 6 & $67, \mathrm{~F}$ & 8 & lung, non-small cell lung cancer & brain, liver & C-6 & $\mathrm{IM}$ & hemiparesis \& voiding difficulty \\
\hline 7 & $54, \mathrm{~F}$ & 16 & lung, adenocarcinoma & brain, liver, adrenal gland & $\mathrm{L}-2$ & IDEM & paraparesis \& voiding difficulty \\
\hline 8 & $14, \mathrm{M}$ & 84 & brain, glioma & no & $\mathrm{T} 11-12$ & $\mathrm{IM}$ & radicular pain \\
\hline 9 & $59, \mathrm{~F}$ & 57 & breast & brain & L2-3 & IDEM & radicular pain \\
\hline
\end{tabular}

* IDEM = intradural extramedullary.

The cases were discussed at the spine tumor board. When the treatment had to be given before the tumor board could discuss the case, rapid consultation was performed for evaluation and treatment decision-making among neurosurgeon, radiation oncologist, and medical oncologist.

\section{Diagnosis and Treatment Planning}

All patients underwent SRS for their IDIM metastasis, with the primary purpose of symptom palliation. Eight patients did not undergo biopsy for the following reasons: 1) the histological diagnosis of primary cancer was already made; 2) MR imaging revealed the typical findings of metastatic spinal cord tumors; and 3) biopsy sampling of the spinal cord carries a potential risk of spinal cord injury. Once the diagnosis and treatment decision were established, image-guided, shaped-beam radiosurgery was performed using the Novalis system (BrainLAB). The positioning devices consist of ExacTrac, an automated patient positioning device, and Novalis Body, an image-guided, target-localization device. Infrared-sensitive markers were placed on the patient's skin before simulation. Vacuum bags were used to immobilize the patient in position. A simulation CT was obtained, with cross-sections measuring 1-3 $\mathrm{mm}$ in thickness without spacing, and the images were sent to the BrainScan planning computer (BrainLAB). The target tumor volume and the critical organs such as the spinal cord were drawn.

The radiosurgery planning used multiple (7-9) intensity-modulated radiation beams to minimize the dose to critical organs, except in 1 patient who was treated with dynamic conformal arc therapy. The average target volume was $3.4 \mathrm{~cm}^{3}$ (range $0.4-12 \mathrm{~cm}^{3}$ ). The median marginal tumor dose was 14 Gy (range 10-16 Gy) in a single session, prescribed to the $90 \%$ isodose line. Multiple lesions were treated simultaneously with individual treat- ment plans. Every effort was made to minimize the spinal cord dose. However, the spinal cord dose exceeded our published guideline of 10 Gy to the $10 \%$ volume that was used for the treatment of epidural compression..$^{15}$ Instead, we allowed a higher radiation dose to the spinal cord in the immediate vicinity of the target tumor. The decision was made on an individual basis by balancing the neurological status and general oncological status of tumor spread with estimated prognosis. For treatment, repositioning was achieved by using an automated infrared tracking system and image fusion between the digitally reconstructed simulation CT image and the orthogonal image.

\section{Posttreatment Follow-Up}

After radiosurgery, patients were followed up with clinical and neurological examinations as well as MR imaging studies. All patients were given steroid treatment at the time of presentation. After radiosurgery, steroid doses were tapered. No chemotherapy was given concurrently with radiosurgery. However, chemotherapy was continued after radiosurgery in patients whose general condition was good enough to undergo additional systemic treatment. Neurological examinations were performed at the time of the diagnosis and at the follow-up clinic visits. The ambulatory grades described by Schiff and O’Neill ${ }^{17}$ were used: Grade 0, asymptomatic; Grade I, patients were able to walk without assistance; Grade II, patients were able to walk with personal or mechanical assistance; Grade III, patients were completely nonambulatory. Clinical response was categorized according to the change in presenting symptoms - that is, improved, unchanged, or worsened. The radiographic response was defined as follows: complete response (disappearance of all target lesions); partial response (30\% decrease in the longest diameter of target lesions); progressive disease 
Stereotactic radiosurgery for spinal intradural metastasis

TABLE 2: Radiosurgery data and clinical outcome in 9 patients with IDIM metastases*

\begin{tabular}{|c|c|c|c|c|c|c|c|c|c|}
\hline \multirow{2}{*}{$\begin{array}{l}\text { Case } \\
\text { No. }\end{array}$} & \multirow[b]{2}{*}{ Modality } & \multirow{2}{*}{$\begin{array}{c}\text { Vol } \\
\left(\mathrm{cm}^{3}\right)\end{array}$} & \multirow{2}{*}{$\begin{array}{l}\text { Dose } \\
\text { (Gy) }\end{array}$} & \multirow{2}{*}{$\begin{array}{l}\text { Prior } \\
\mathrm{Tx}\end{array}$} & \multicolumn{2}{|c|}{ Ambulation Grade $\dagger$} & \multirow{2}{*}{$\begin{array}{c}\text { Clinical } \\
\text { Response }\end{array}$} & \multirow{2}{*}{$\begin{array}{c}\text { Radiological } \\
\text { Response }\end{array}$} & \multirow{2}{*}{$\begin{array}{c}\text { Survival } \\
\text { (mos) }\end{array}$} \\
\hline & & & & & Pre-SRS & Post-SRS & & & \\
\hline 1 & IMRT & 0.4 & 16 & no & 0 & 0 & improved & CR & 5.2 \\
\hline \multirow[t]{2}{*}{2} & IMRT & 0.6 & 16 & no & III & $\|$ & improved & PR & \\
\hline & IMRT & 3.2 & 16 & no & III & III & worsened & PD & 5.9 \\
\hline 3 & IMRT & 0.7 & 14 & no & III & III & unchanged & SD & 2.2 \\
\hline 4 & IMRT & 1.0 & 14 & no & I & I & improved & NA & 5.2 \\
\hline 5 & IMRT & 1.8 & 12 & EBRT & I & I & improved & PR & 19.4 \\
\hline 6 & IMRT & 3.7 & 16 & op & ॥ & $\|$ & improved & SD & 5.2 \\
\hline 7 & IMRT & 1.8 & 14 & no & I & I & improved & CR & 5.5 \\
\hline 8 & dynamic conformal & 12.0 & 10 & EBRT & I & I & improved & SD & 14.0 \\
\hline 9 & IMRT & 3.6 & 14 & no & 0 & 0 & improved & PR & 4.5 (alive) \\
\hline
\end{tabular}

(20\% increase in the longest diameter of target lesions); stable disease (small changes that do not meet the aforementioned criteria). ${ }^{6}$ Survival was calculated from the time of SRS until death or the last follow-up visit. The actual overall survival was calculated according to the Kaplan-Meier method.

\section{Results}

\section{Patient Characteristics}

From the database of 652 spine radiosurgery procedures performed between 2001 and 2008, 9 patients were identified who had IDIM metastasis treated with SRS. There were a total of 11 lesions (1 of which was not evaluable); 4 intradural extramedullary lesions and 7 intramedullary lesions. Of the 9 patients, 6 were female and 3 were male. The mean age at presentation was 50 years, with a range of $14-71$ years. The common primary tumor sites were breast in 3 patients $(33 \%)$, followed by lung in 2 patients $(22 \%)$, brain in $2(22 \%)$, kidney in 1 $(11 \%)$, and skin in $1(11 \%)$. The IDIM metastasis was predominantly located in the cervical spinal cord (7 [64\%] of 11 lesions) followed by the thoracic cord (2 [18\%] of 11 lesions) and conus medullaris (1 [9\%] of 11 lesions). The mean time from the primary cancer diagnosis to the development of IDIM metastasis was 4 years, with a range of 8 months-14 years. In 1 patient (Case 6), IDIM was the initial presentation in the course of the cancer. All patients had simultaneous metastatic lesions in the brain at the time of presentation, and among them, 2 patients also had progressive systemic metastases. The patient characteristics are shown in Table 1.

\section{Radiosurgery Parameters}

Radiosurgery information and clinical course are summarized in Table 2. Eight patients underwent IMRT and 1 had dynamic conformal arc therapy. Among the 8 cases treated with IMRT, in 7 cases we used the coplanar method and in 1 we used the noncoplanar method, using 7-9 beams. The median marginal radiosurgery dose was 14 Gy, with a range of 10-16 Gy, prescribed to the $90 \%$ isodose line. The mean tumor volume was $3.4 \mathrm{~cm}^{3}$, with a range of $0.4-12 \mathrm{~cm}^{3}$. The entire target volume was covered by the prescription dose. Stereotactic radiosurgery was performed as the sole initial treatment in 6 patients, 2 (Cases 5 and 8) underwent SRS following EBRT, and 1 (Case 6) received SRS following the resection with which the initial diagnosis was made.

\section{Clinical Improvement}

The presenting symptoms were improved in $8(80 \%)$

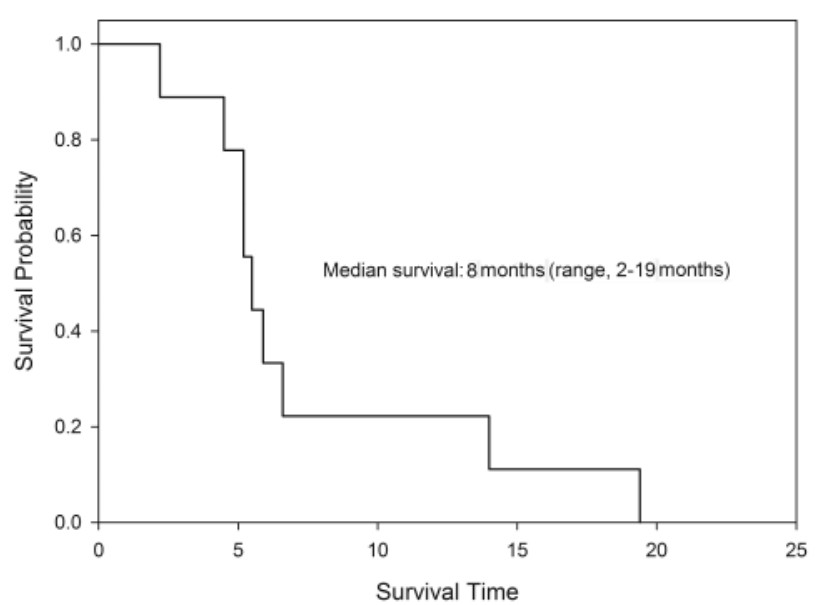

FIG. 1. Kaplan-Meier survival curve showing survival times in 9 patients with IDIM metastases. 

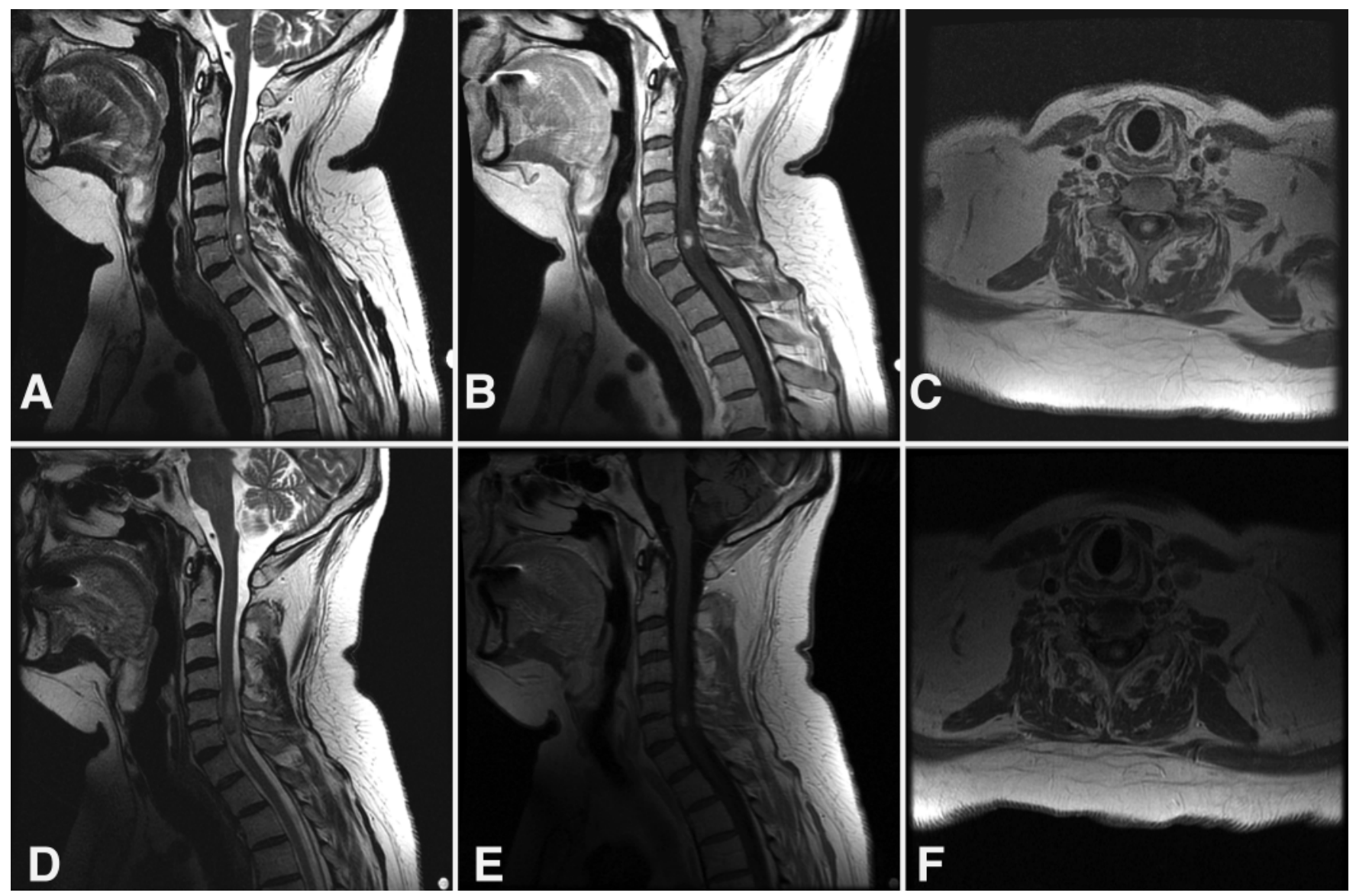

FIG. 2. Case 1. Preradiosurgery $(A-C)$ and postradiosurgery $(D-F)$ MR imaging studies obtained in a patient with melanoma. Before radiosurgery, the T2-weighted $(A)$ and T1-weighted with contrast $(B$ and $C$ ) images show a heterogeneously enhancing intramedullary lesion with hemorrhage at the level of C-6. At 3 months postradiosurgery, resolution of the enhancing lesion $(E$ and $F)$, with remaining hemosiderin, was shown. There is no other spinal cord abnormality on the T2-weighted (D) image.

of 10 evaluable lesions, unchanged in 1 case, and worsened in 1. Pain and numbness responded to radiosurgery promptly in all cases. Before radiosurgery, 7 patients were ambulatory, and 5 of them had some gait disturbance. After radiosurgery, all these patients maintained their walking ability with or without assistance. One patient had prompt recovery of ambulation after radiosurgery (Case 2), although this individual later developed progressive metastases at other spine segments and became nonambulatory. There were 2 patients who were nonambulatory before radiosurgery, and afterward they were still nonambulatory; clinical responses after radiosurgery are summarized in Table 2 . The overall median survival after radiosurgery was 8 months (range 2-19 months). Eight of the 9 patients died during the follow-up period. The Kaplan-Meier survival curve is shown in Fig. 1.

\section{Radiographic Response}

Follow-up images were available in 9 lesions in 8 patients. One patient was not able to undergo MR imaging studies because of claustrophobia. There were 2 cases with complete response (22\%), 3 cases with partial response (33\%), 3 cases with stable disease $(33 \%)$, and 1 case with progressive disease $(11 \%)$. Overall tumor control was achieved in 8 cases $(89 \%)$. An example of mela- noma intramedullary metastasis with hemorrhage at C-6 is shown in Fig. 2. This patient had clinical symptoms of right-sided deltoid palsy, with a motor grade of IV/V and numbness in the arm and leg on the same side. Radiosurgery was given (16 Gy), and 3 months after treatment the patient's neurological symptoms disappeared. Follow-up MR imaging showed resolution of the enhancing lesion, with remaining hemosiderin. Another example of breast cancer with intradural metastsis is illustrated. Figure 3 shows an enhancing intradural mass at the level of L2-3, occupying the central and right lateral aspect of the thecal sac. This patient was treated with radiosurgery (14 Gy) to the lesion. The patient experienced symptom improvement, with radicular pain in her right leg. Follow-up MR imaging obtained 3 months after radiosurgery showed a significant reduction of the intradural mass.

There was no radiation toxicity detected clinically during the follow-up period. There was no abnormality within the spinal cord on T2-weighted or contrast-enhancing images that was suggestive of radiation effect.

\section{Discussion}

An IDIM metastasis is one of the most severe complications of systemic cancer. ${ }^{3,18}$ Optimal treatment of an IDIM lesion is still challenging, although IDIM has been 

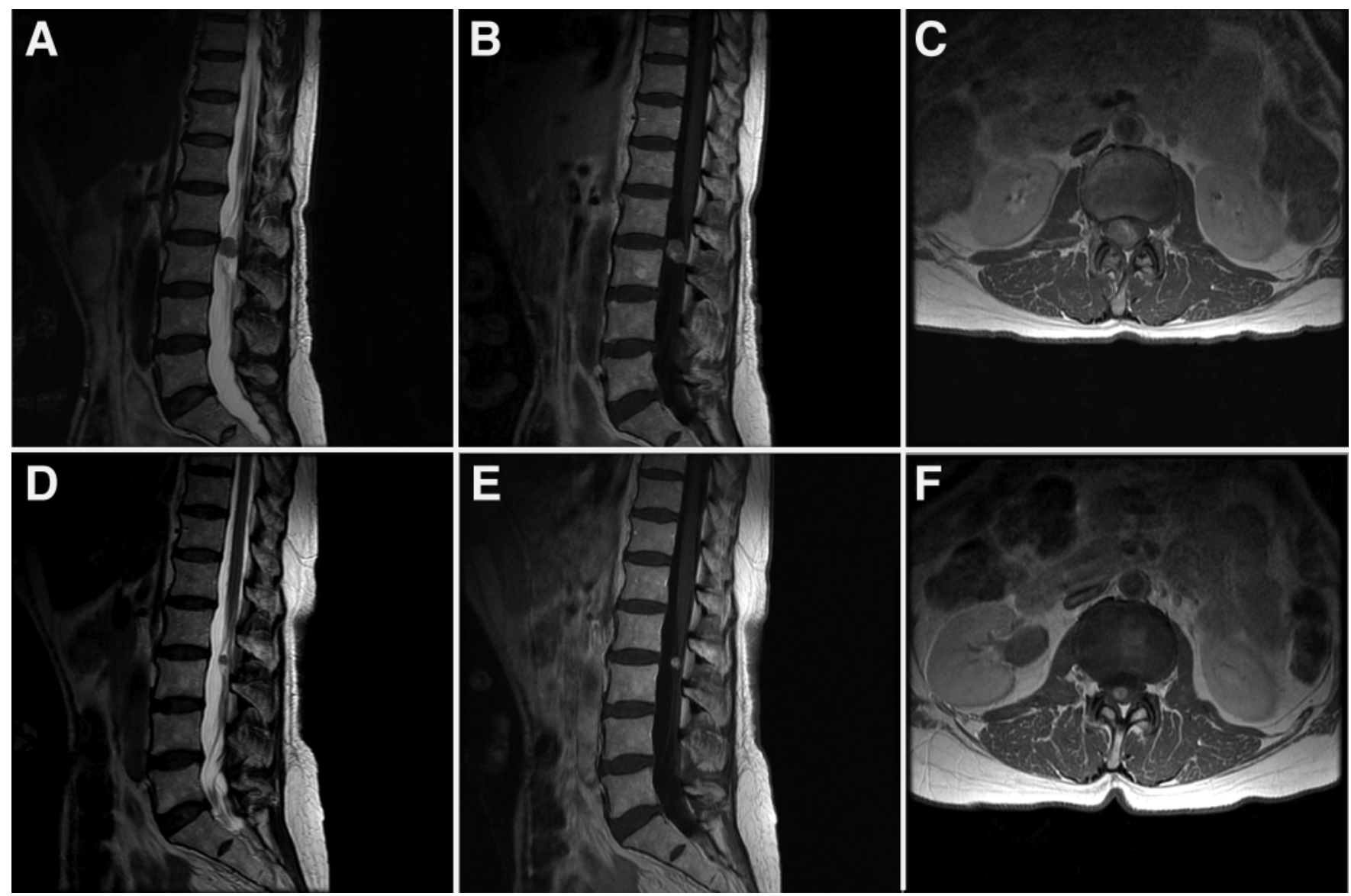

FIG. 3. Case 9. Preradiosurgery (A-C) and postradiosurgery (D-F) MR imaging studies obtained in a patient with breast cancer. Before radiosurgery, T2-weighted $(A)$ and T1-weighted with contrast ( $B$ and $C$ ) images show an enhancing intradural mass at the level of L2-3, occupying the central and right lateral aspect of the thecal sac. At 3 months postradiosurgery, significant interval diminution of the intradural mass was noted on T2-weighted (D) and T1-weighted with contrast (E and F) images.

more readily detected with MR imaging. There is a lack of prospective randomized trials, and retrospective studies included small numbers of patients with limited tumor types. ${ }^{3,8,18}$ As the overall survival has increased in patients with malignancies, quality of life has become important for both definitive and palliative treatment ${ }^{16}$ Because incapacitating pain, paralysis, sensory loss, and loss of sphincter function are known to occur in untreated patients, effective treatments are necessary to improve the quality of life. ${ }^{2}$ Treatment options for IDIM metastasis may include resection, EBRT, systemic treatment with chemotherapy, or hormone therapy, depending on the cell type. These treatments are generally limited and, therefore, have been used empirically and on a case-by-case basis. Among the available therapeutic options, fractionated radiotherapy has been the most effective treatment when the patients are not neurologically compromised..$^{3,8,17}$ However, in the case of fractionated radiotherapy, a significant amount of normal tissue, including healthy spinal cord, is included within the treatment volume. Practically, patients should visit the clinic for a protracted course of radiotherapy despite their critically ill status. Surgery has been considered in selected patients with a reasonable life expectancy and rapid neurological deterioration, or when tissue diagnosis is lacking. ${ }^{2,8-10}$ Although the resection rate has been im- proved with technical advancement, the risk of postoperative functional impairment is still high, especially in intramedullary metastases. ${ }^{9}$ In addition, a significant group of patients are not candidates for a major surgery or anesthesia due to their poor general condition. Chemotherapy is a reasonable treatment option when the primary tumor is likely to be chemosensitive. Intrathecal chemotherapy failed to show any objective response. ${ }^{11}$

The result of our experience with radiosurgery for IDIM metastasis is encouraging. Although this study was retrospective, our findings demonstrated that SRS is a safe and effective modality for symptom control and neurological stabilization. The population characteristics were similar to those in previous reports. In accordance with previous studies, lung and breast cancer accounted for $55 \%$ of all cases. ${ }^{8,17}$ The cervical cord has been reported as being the most common site, and this is also consistent with our series. ${ }^{7}$ Because IDIM occurs in advanced stages of cancer, it often accompanies brain and systemic metastasis. ${ }^{2,7,9}$ In the present study, brain metastasis was noted in all patients, and other metastases in 2 patients. In our series, all patients suffered from pain and neurological dysfunction.

In this study, we identified several advantages of SRS in patients with IDIM metastasis. First, SRS was effective in tumor control and neurological improvement. Present- 
ing symptoms were relieved in $80 \%$ of cases, and overall tumor control was achieved in $89 \%$ of patients. Although 8 patients $(89 \%)$ died during the study period, ambulatory status was maintained for most of their remaining life. Because SRS is able to deliver a highly conformal and large radiation dose to a localized tumor while minimizing the radiation dose to the normal tissue, better tumor control might be achieved. Second, SRS in patients with IDIM metastasis was safe. . $^{13,14,16}$ No single complication was noted in relation to the SRS procedure in the present study. Of note is that the survival of these patients was so short that the potential long-term radiation-induced myelopathy was not manifested. We have carefully observed all the spine radiosurgery cases regarding the spinal cord tolerance of single-dose radiosurgery. In the series, we demonstrated that 10 Gy to the $10 \%$ volume of the spinal cord volume (defined as $6 \mathrm{~mm}$ above and below the radiosurgery target volume) is safe..$^{15}$ In the radiosurgery of IDIM targets, there was a small volume of spinal cord that may have been treated at higher doses. Nevertheless, there were no clinical or radiographic abnormalities, although longer follow-up may be necessary to assess the long-term radiation-induced complications. The precision for a given isocenter between the simulation and the actual treatment position was reported to be within $1.36 \pm$ $0.11 \mathrm{~mm} .^{13,14,16}$ The effort of pursuing this extreme precision can minimize the chance of complication. Third, SRS was convenient in all patients. A single or minimal number of hospital visits were more convenient for this group of critically ill patients and for their caregivers. Nonetheless, SRS also has limitations, and needs further studies to define its role in the treatment of IDIM metastasis.

\section{Conclusions}

Despite the fact that this was a small series of patients with IDIM metastasis who had limited treatment options, SRS appears to be an effective and safe treatment for patients with these lesions.

\section{Disclaimer}

The authors have no personal or institutional financial interest in any of the materials described in the current study.

\section{References}

1. Benzil DL, Saboori M, Mogilner AY, Rocchio R, Moorthy CR: Safety and efficacy of stereotactic radiosurgery for tumors of the spine. J Neurosurg 101 (3 Suppl):413-418, 2004

2. Connolly ES Jr, Winfree CJ, McCormick PC, Cruz M, Stein BM: Intramedullary spinal cord metastasis: report of three cases and review of the literature. Surg Neurol 46:329-338, 1996

3. Dam-Hieu P, Seizeur R, Mineo JF, Metges JP, Meriot P, Simon $\mathrm{H}$ : Retrospective study of 19 patients with intramedullary spinal cord metastasis. Clin Neurol Neurosurg 111:10-17, 2009
4. De Salles AA, Pedroso AG, Medin P, Agazaryan N, Solberg T, Cabatan-Awang C, et al: Spinal lesions treated with Novalis shaped beam intensity-modulated radiosurgery and stereotactic radiotherapy. J Neurosurg 101 (3 Suppl):435-440, 2004

5. Finn MA, Vrionis FD, Schmidt MH: Spinal radiosurgery for metastatic disease of the spine. Cancer Control 14:405-411, 2007

6. Hitchins RN, Bell DR, Woods RL, Levi JA: A prospective randomized trial of single-agent versus combination chemotherapy in meningeal carcinomatosis. J Clin Oncol 5:16551662,1987

7. Kalayci M, Cağavi F, Gül S, Yenidünya S, Açikgöz B: Intramedullary spinal cord metastases: diagnosis and treatment-an illustrated review. Acta Neurochir (Wien) 146:1347-1354, 2004

8. Lee SS, Kim MK, Sym SJ, Kim SW, Kim WK, Kim SB, et al: Intramedullary spinal cord metastases: a single-institution experience. J Neurooncol 84:85-89, 2007

9. Mut M, Schiff D, Shaffrey ME: Metastasis to nervous system: spinal epidural and intramedullary metastases. J Neurooncol 75:43-56, 2005

10. Ogino M, Ueda R, Nakatsukasa M, Murase I: Successful removal of solitary intramedullary spinal cord metastasis from colon cancer. Clin Neurol Neurosurg 104:152-156, 2002

11. Orlando L, Curigliano G, Colleoni M, Fazio N, Nole F, Martinelli $\mathrm{G}$, et al: Intrathecal chemotherapy in carcinomatous meningitis from breast cancer. Anticancer Res 22:30573059, 2002

12. Rock JP, Ryu S, Yin FF: Novalis radiosurgery for metastatic spine tumors. Neurosurg Clin N Am 15:503-509, 2004

13. Rock JP, Ryu S, Yin FF, Schreiber F, Abdulhak M: The evolving role of stereotactic radiosurgery and stereotactic radiation therapy for patients with spine tumors. J Neurooncol 69:319334,2004

14. Ryu S, Fang Yin F, Rock J, Zhu J, Chu A, Kagan E, et al: Image-guided and intensity-modulated radiosurgery for patients with spinal metastasis. Cancer 97:2013-2018, 2003

15. Ryu S, Jin JY, Jin R, Rock J, Ajlouni M, Movsas B, et al: Partial volume tolerance of the spinal cord and complications of single-dose radiosurgery. Cancer 109:628-636, 2007

16. Ryu S, Jin R, Jin JY, Chen Q, Rock J, Anderson J, et al: Pain control by image-guided radiosurgery for solitary spinal metastasis. J Pain Symptom Manage 35:292-298, 2008

17. Schiff D, O’Neill BP: Intramedullary spinal cord metastases: clinical features and treatment outcome. Neurology 47:906912, 1996

18. Waki F, Ando M, Takashima A, Yonemori K, Nokihara H, Miyake M, et al: Prognostic factors and clinical outcomes in patients with leptomeningeal metastasis from solid tumors. J Neurooncol 93:205-212, 2009

19. Whang CJ, Yee GT, Choi CY, Sohn MJ, Lee DJ: First experience in using Novalis shaped beam radiosurgery in Korea. J Neurosurg 101 (3 Suppl):341-345, 2004

\footnotetext{
Manuscript submitted August 15, 2009.

Accepted September 3, 2009.

Address correspondence to: Samuel Ryu, M.D., Department of Radiation Oncology and Neurosurgery, Henry Ford Health System, 2799 West Grand Boulevard, Detroit, Michigan 48202. email: sryu1@hfhs.org.
} 